\title{
A kind of differential sliding mode controller design for pitch channel simplified model of hypersonic aircraft
}

\author{
Guoqiang Liang $^{\mathrm{a}}$ and Guangbin Wu \\ Department of control engineering, Naval aeronautical and astronautical University \\ Yanti, 264001 \\ aliangguoqiang1024@126.com
}

Keywords: hypersonic aircraft; stability; control; free flying; numerical simulation

\begin{abstract}
A kind of differential type sliding mode control law was proposed to improve the robustness of a kind of simplified pitch channel model of hypersonic aircraft which is widely discussed recently. The advantage of sliding mode control is that it has differential item which can provide a special damping for flying system. With the increasing of damping ratio, the stable margin of the who flying system can be greatly increased. Also, to prove the stability of the whole system, two Lyapunov functions were constructed and all signals of close system are proved to be stable. At last, detailed simulation was done to testify the effectiveness of the proposed method.
\end{abstract}

\section{Introduction}

The control problem of hypersonic vehicle has aroused the interest of many researchers in the world. The hypersonic vehicle generally refers to aircraft with flight velocity of more than 5 Maher in the atmosphere. For sliding mode variable structure control ( SMVSC) system, the so-called "variable structure" is essentially refers to the discontinuous nonlinear shear of system's internal feedback controller structure. Sliding mode control has been widely used in flight control system, Shtessel ${ }^{[1-5]}$ et al. use sliding mode control technology for reusable launch vehicle X33 to design the flight control system, and good control effect is achieved, and based on that, control system performance was further improved through the introduction of sliding mode observer ${ }^{[6-8]}$. But the difficult problem of high speed control lies in its model complexity, which leads to lack of stable margin for common methods. So this paper adopts a kind of differential sliding mode control method to improve its damping and increase algorithm stability.

\section{Model Description}

A kind of hypersonic vehicle can be simplified as below differential equations to described its pitch channel movement:

$$
\begin{gathered}
\dot{V}=\frac{T \cos \alpha-D}{m}-g \sin \gamma \\
\dot{\gamma}=\frac{L+T \sin \alpha}{m V}-\frac{g \cos \gamma}{V}
\end{gathered}
$$




$$
\begin{gathered}
\dot{\alpha}=q-\dot{\gamma} \\
\dot{q}=\frac{M}{I} \\
\dot{h}=V \sin \gamma \\
\ddot{\eta}=-2 \varsigma \omega_{n} \dot{\eta}-\omega_{n}^{2} \eta+\omega_{n}^{2} \eta_{c}, \omega_{n}=5, \varsigma=0.7
\end{gathered}
$$

Where aero coefficient is identified as

$$
\begin{gathered}
L=\bar{q} S C_{L}, C_{L}=C_{L}^{\alpha} \alpha+C_{L}^{\delta} \delta+C_{L}^{0}, \quad T=\bar{q} S C_{T} \\
D=\bar{q} S C_{D}, C_{D}=C_{d}^{\alpha^{2}} \alpha^{2}+C_{D}^{\alpha} \alpha+C_{D}^{\delta^{2}} \delta^{2}+C_{D}^{\delta} \delta+C_{D}^{0} \\
M=\bar{q} S \bar{c}\left[C_{M \alpha}+C_{M \delta}+C_{M q}\right], C_{M \alpha}=C_{M \alpha}^{\alpha^{2}} \alpha^{2}+C_{M \alpha}^{\alpha} \alpha+C_{M \alpha}^{0}, \\
C_{M \delta}=C_{e}\left(\delta_{e}-\alpha\right), \\
C_{M \alpha}=10^{-4}\left(0.06-e^{-M_{a} / 3}\right)\left(-2 \alpha^{2}+120 \alpha-1\right) \\
C_{M q}=\frac{\bar{c} q}{2 V}\left(-0.025 M_{a}+1.37\right)\left(-0.0021 \alpha^{2}+0.0053 \alpha-0.23\right) \\
C_{M \delta}=0.0292(\delta-\alpha) \\
C_{L}=\alpha\left(0.493+1.91 / M_{a}\right) \\
C_{D}=0.0082\left(171 \alpha^{2}+1.15 \alpha+2\right)\left(0.0012 M_{a}^{2}-0.054 M_{a}+1\right) \\
C_{T}=\left\{\begin{array}{l}
38\left[1-164\left(\alpha-\alpha_{0}\right)^{2}\right]\left(1+17 / M_{a}\right)(1+0.15) \eta, \eta<1 \\
38\left[1-164\left(\alpha-\alpha_{0}\right)^{2}\right]\left(1+17 / M_{a}\right)(1+0.15 \eta), \eta<1
\end{array}\right\}
\end{gathered}
$$

And $V$ is speed, $\gamma$ is the speed angle, $\alpha$ is attack angle, $Q$ is the attitude angle speed, $h$ is the height.

$\phi$ is the oil supplying factor, $\delta_{c}$ is the duck wing and $\delta_{e}$ is the lift rudder.

\section{Sliding mode controller design}

Define error variable as $e=\alpha-\alpha^{d}$, and choose a common integral sliding mode variable as

$$
S=\dot{e}+c_{1} e
$$

Solve the derivative of sliding mode as

$$
\dot{s}=\ddot{e}+c_{1} \dot{e}
$$

Then it can be transformed as 


$$
\dot{s}=\ddot{\alpha}+c_{1} \dot{\alpha}
$$

Then

$$
\dot{s}=\dot{q}-\ddot{\gamma}+c_{1} q-c_{1} \dot{\gamma}
$$

By using the information of above model, it can be written as

$$
\dot{s}=\frac{M}{I}-\ddot{\gamma}+c_{1} q-c_{1}\left(\frac{L+T \sin \alpha}{m V}-\frac{g \cos \gamma}{V}\right)
$$

And it can be rewritten as

$$
\dot{s}=\frac{\left(\bar{q} S \bar{c}\left[C_{M \alpha}+C_{M \delta}+C_{M q}\right]\right)}{I}-\ddot{\gamma}+c_{1} q-c_{1}\left(\frac{L+T \sin \alpha}{m V}-\frac{g \cos \gamma}{V}\right)
$$

Finally, we get

$$
\begin{aligned}
& \dot{s}=\frac{\left(\overline { q } S \overline { c } \left[10^{-4}\left(0.06-e^{-M_{a} / 3}\right)\left(-2 \alpha^{2}+120 \alpha-1\right)+0.0292(\delta-\alpha)\right.\right.}{I} \\
& \left.\left.+\frac{\bar{c} q}{2 V I}\left(-0.025 M_{a}+1.37\right)\left(-0.0021 \alpha^{2}+0.0053 \alpha-0.23\right)\right]\right) \\
& -\ddot{\gamma}+c_{1} q-c_{1}\left(\frac{L+T \sin \alpha}{m V}-\frac{g \cos \gamma}{V}\right)
\end{aligned}
$$

And consider that the variable $L$ can also be written as functions of attack angle and actuator, then it can be written as follows:

$$
\begin{aligned}
& \dot{s}=\frac{\left(\overline { q } S \overline { c } \left[10^{-4}\left(0.06-e^{-M_{a} / 3}\right)\left(-2 \alpha^{2}+120 \alpha-1\right)+0.0292(\delta-\alpha)\right.\right.}{I} \\
& \left.\left.+\frac{\bar{c} q}{2 V I}\left(-0.025 M_{a}+1.37\right)\left(-0.0021 \alpha^{2}+0.0053 \alpha-0.23\right)\right]\right) \\
& -\ddot{\gamma}+c_{1} q-c_{1}\left(\frac{T \sin \alpha}{m V}-\frac{g \cos \gamma}{V}\right)-\frac{c_{1} \bar{q} S\left(C_{L}^{\alpha} \alpha+C_{L}^{\delta} \delta+C_{L}^{0}\right)}{m V}
\end{aligned}
$$

And define new variables as follows

$$
\begin{gathered}
l_{2}(\alpha, q, e)=\frac{\left(\bar{q} S \bar{c}\left[10^{-4}\left(0.06-e^{-M_{a} / 3}\right)\left(-2 \alpha^{2}+120 \alpha-1\right)-0.0292 \alpha\right)\right.}{I} \\
\left.\left.+\frac{\bar{c} q}{2 V I}\left(-0.025 M_{a}+1.37\right)\left(-0.0021 \alpha^{2}+0.0053 \alpha-0.23\right)\right]\right) \\
-\ddot{\gamma}+c_{1} q-c_{1}\left(\frac{T \sin \alpha}{m V}-\frac{g \cos \gamma}{V}\right)-\frac{c_{1} \bar{q} S\left(C_{L}^{\alpha} \alpha+C_{L}^{0}\right)}{m V} \\
l_{1}=\frac{\bar{q} S \bar{c} 0.0292}{I}-\frac{c_{1} \bar{q} S C_{L}^{\delta}}{m V}
\end{gathered}
$$

Then the derivative of sliding mode can be written as

$$
\dot{s}=l_{1} \delta+l_{2}(\alpha, q, e)
$$

Then the sliding mode control law can be designed as 


$$
\delta=-\operatorname{sign}\left(l_{1}\right)\left[k_{1} s+k_{2} \frac{s}{|s|+k_{3}}+k_{4} s^{1 / 3}+k_{5} \int s d t\right]
$$

Where $k_{i}>0$, and assume there exist a big enough positive constant such $k_{6}$ and $k_{7}$ that

$$
\left|l_{2}(\alpha, q, e)\right|<k_{6}|s|+k_{7}
$$

Choose a Lyapunov function as

$$
V=\frac{1}{2} s^{2}+\frac{k_{5}\left|l_{1}\right|}{2}\left(\int s d t\right)^{2}
$$

Then solve its derivative, it satisfies

$$
\begin{aligned}
& \dot{V}=s \dot{s}+k_{5}\left|l_{1}\right|\left(s \int s d t\right) \\
& =l_{1} \delta s+s l_{2}(\alpha, q, e)+k_{5}\left|l_{1}\right|\left(s \int s d t\right)
\end{aligned}
$$

And it can be written as

$$
\dot{V}=-\left|l_{1}\right|\left[k_{1} s^{2}+k_{2} \frac{s^{2}}{|s|+k_{3}}+k_{4} s^{4 / 3}+k_{5} s \int s d t\right]+s l_{2}(\alpha, q, e)+k_{5}\left|l_{1}\right|\left(s \int s d t\right)
$$

With the help of inequality method, it can be rewritten as

$$
\begin{aligned}
& \dot{V} \leq-\left|l_{1}\right|\left[k_{1} s^{2}+k_{2} \frac{s^{2}}{|s|+k_{3}}+k_{4} s^{4 / 3}+k_{5} s \int s d t\right]+k_{6} s^{2}+k_{7}|s|+k_{5}\left|l_{1}\right|\left(s \int s d t\right) \\
& =-\left|l_{1}\right|\left[k_{1} s^{2}+k_{2} \frac{s^{2}}{|s|+k_{3}}+k_{4} s^{4 / 3}\right]+k_{6} s^{2}+k_{7}|s|
\end{aligned}
$$

So it is easy to choose big enough parameters $k_{1}, k_{2}$ and $k_{3}$ such that

$$
\dot{V} \leq 0
$$

Then according to Lyapunov theory, we can prove $s \rightarrow 0$.

Since $c_{1}>0$, then it has

$$
\dot{e}=-c_{1} e
$$

Choose another Lyapunov function as

$$
V_{a}=e^{2} / 2
$$

Then it holds

$$
\dot{V}_{a} \leq 0
$$

Then according to Lyapunov theory, we have $e \rightarrow 0$, then finally we can prove that the system is stable. 


\section{Numerical Simulation and result analysis}

Set model parameters as

$$
\begin{gathered}
I_{x x}=-7.1 * 10^{-5} \mathrm{~m}^{2}+19.1 \mathrm{~m}-59430 \\
I_{y y}=-8.03 * 10^{-4} \mathrm{~m}^{2}+219.74 m-1690000 \\
I_{z z}=-8.03 * 10^{-4} \mathrm{~m}^{2}+219.74 m-1690000 \\
I_{y y 0}=1.23 * 10^{7}, v_{s}=3.017 * 10^{2}, \rho_{a}=1.84 * 10^{2}, \\
g_{a}=9.7147, \quad h=30000 \\
V=4525, \eta_{c}=0.15662
\end{gathered}
$$

With above model and air coefficients and set initial condition as above paragraph and write a program with $\mathrm{m}$ language in Matlab software, then the simulation can be done with above control law where desired value of attack angle is set as 4 degree, and simulation results can be shown as following figures.

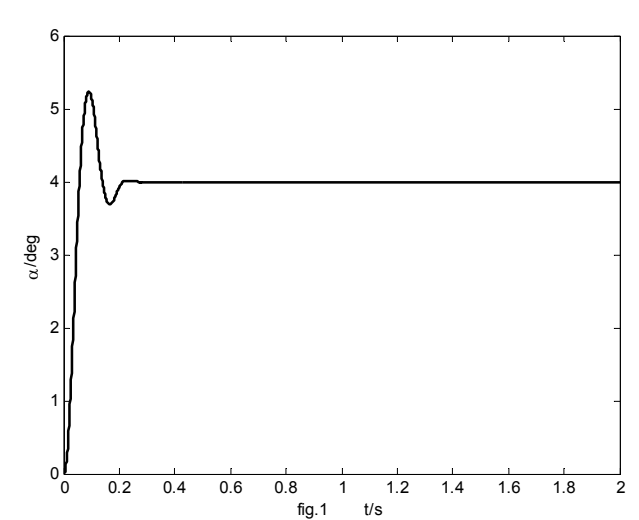

Fig 1 The curve of attack angle

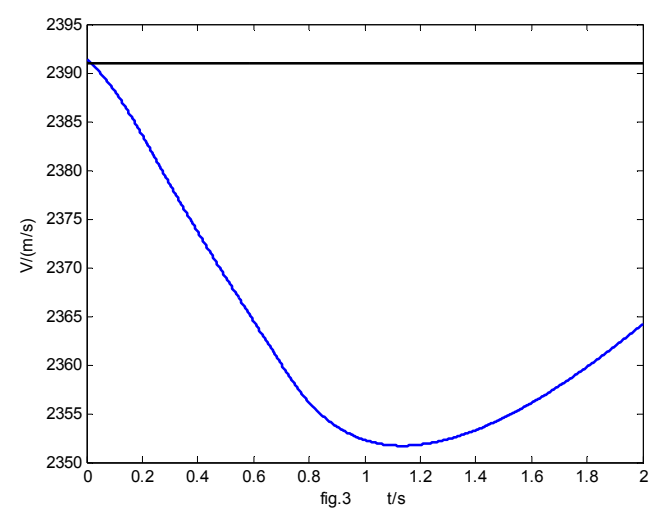

Fig 3 The curve of speed

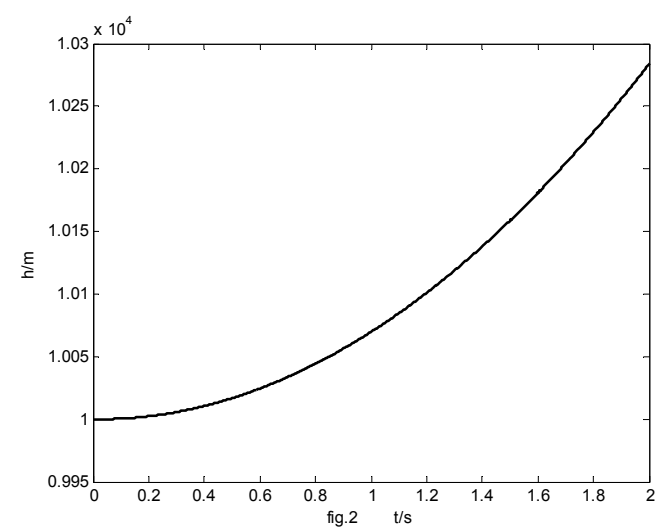

Fig 2 The curve of the height

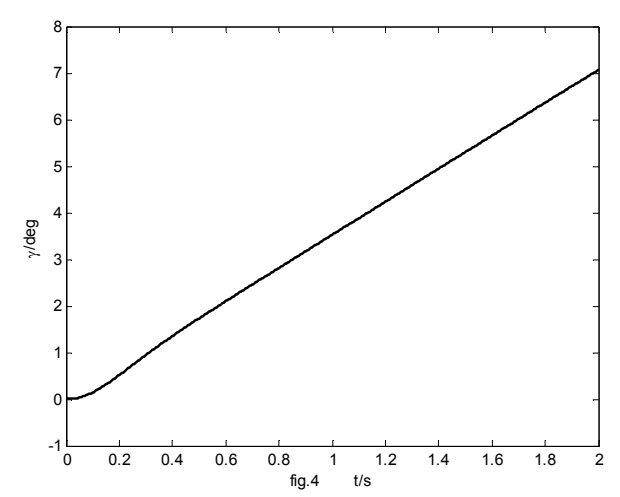

Fig 4 The curve of speed angle

From the above simulation results, we can see that the method provided in this paper has a good rapidity, the angle of attack response time is about $0.1 \mathrm{~s}$. The curve of thrust has a partial time to keep on zero, which is mainly due to the small speed error make the fuel supply factor to be negative. 


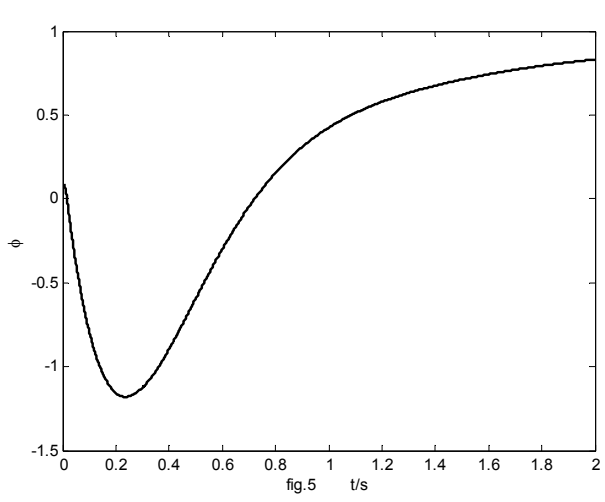

Fig 5 The curve of oil supplying factor

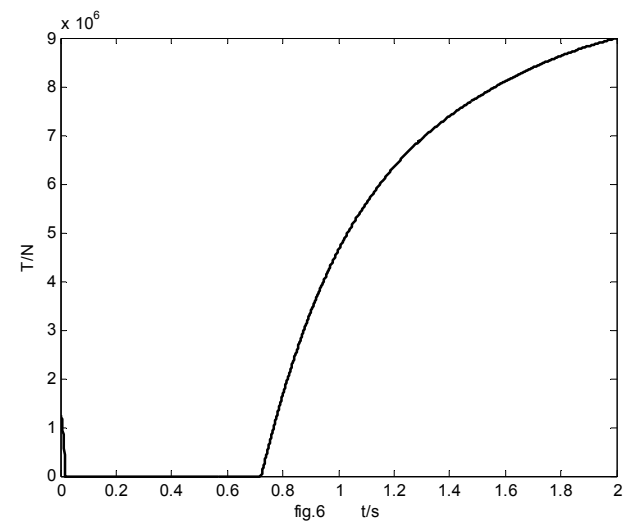

Fig 6 The curve of thrust

\section{Conclusion}

In this paper, a class of differential sliding mode control method is proposed to solve the tracking problem of attack angle for a class of simplified pitch channel model of hypersonic vehicle. Due to the introduction of the differential signal, the system can provide sufficient damping, and the sliding mode control itself has a strong robustness, so the structure of the method is reasonable. By constructing the Lyapunov function, the system is guaranteed to be bounded and stable. Finally, the simulation results show that the method is effective, and it has a good rapidness and good performance.

\section{References}

[1]Buschek H and Calise A J.Hypersonic flight control system design using fixed order robust controllers[A].In:AIAA Guidance,Navigation and Control Conference[C].Chattanooga,TN, AIAA 95-6062

[2]Buschek H, Calise A J. Uncertainty modeling and fixed-order controller design for a hypersonicvehicle model. Journal of Guidance, Control and Dynamics, 1997, 20(1): 42-48.

[3]Heller M and Sachs G.Flight dynamics and robust control of a hypersonic test vehicle with ramjet propulsion[A].In:AIAA International Space Planes and Hypersonic Systems and Technologies Conference[C].Norfolk,VA,AIAA 98-1521

[4]M. Ohno, Y. Yamaguchi, T. Hata, M. Takahama, Robust flight control law design for an automatic landing flight experiment [J], Control Engineering Practice, 1999, 7(9):1143-1152

[5]Naidu S D,Banda S S and Buffington J L.Unified approach to $\mathrm{H}_{2}$ and $\mathrm{H}_{\infty}$ optimal control of a hypersonic vehicle[A].In:Proceedings of the American control conference[C].San Diego, California,1999, vol.4:2737-2741.

[6]Christopher I. Marrison. synthesis of robust control systems for a hypersonic aircraft decision and contro1. IEEE, 1994。4:3324-3329

[7]E. mooij. linear quadratic regulator reentry control-performance assessment using a taguchi approach. AIAA, international space planes and hypersonic systems and technologies conference. 1998: 665-677.

[8]Kevin. P. Groves, Andrea. Serranti, Stephen. Yurkovich. Anti-Windup Control for an Air-breathing Hypersonic Vehicle Model. AIAA, 2006-6557: 1-14. 